\title{
Intercalated Disk
}

National Cancer Institute

\section{Source}

National Cancer Institute. Intercalated Disk. NCI Thesaurus. Code C32819.

The intercellular structures including the fascia adherens, gap junctions and macula adherens that connect sing le cardiac muscle cells to allow for synchronized contractions. 